\title{
Computer Assisted Language Learning (CALL) Software: Evaluation of its Influence in a Language Learning Process
}

\author{
Ugochukwu C. Okonkwo
}

DOI: http://dx.doi.org/10.4314/ujah.v12i1.4

\section{Abstract}

Evaluating the nature and extent of the influence of Computer Assisted Language Learning (CALL) on the quality of language learning is highly problematic. This is owing to the number and complexity of interacting variables involved in setting the items for teaching and learning languages. This paper identified and characterised features and processes through which computer assisted language learning impacts upon learning language. It offers a framework for analysing the effects of computer assisted language learning in combination with other factors which may enhance or ameliorate the positive impact of it in the classroom and beyond.

\section{Introduction}

Recent research has shown that human language is much more complex than it was previously thought. So development and usage of computer assisted language learning often requires the services of members of interdisciplinary teams. Hence, computer assisted language learning draws upon the involvement of computer scientists, engineers, linguists, experts in artificial intelligence, cognitive psychologists, mathematicians, logicians, amongst others. (Ellis 2004)

Computer assisted language learning (CALL) is a form of computer-based learning which carries two important features: bidirectional learning and individualized learning. It 
is not a method. The focus of CALL is learning, and not teaching. CALL materials are used in teaching to facilitate the language learning process. It is a student-centered learning material, which promotes self-paced learning. CALL has also been known by several other terms such as technology-enhanced language learning (TELL), computerassisted language instruction (CALI) and computer-aided language learning, but the field is the same. The philosophy of CALL is that the lessons should allow the learners to learn on their own using structured and/or unstructured interactive lessons. Structured interactive lessons is the type that had been systematically arranged previously and has scaled through the performance measure, while unstructured interactive lessons is an impromptu lessons that are formulated and are yet to be subjected to performance measure.

\section{The Use of CALL for the Four Skills}

A number of studies have been done concerning how the use of CALL affects the development of language learners' four skills (listening, speaking, reading and writing). Most report significant gains in reading and listening and most CALL programs are geared toward these receptive skills because of the current state of computer technology in linguistics. However, most reading and listening software is based on drills (Domingo, 2007). Gains in writing skills have not been as impressive as computers cannot assess this well (Stepp, 2002).

However, using current CALL technology, even with its current limitations, for the development of speaking abilities, has gained much attention. There has been some success in using CALL, in particular computer-mediated communication, to help speaking skills closely linked to 
"communicative competence" (ability to engage in meaningful conversation in the target language) and provide controlled interactive speaking practice outside the classroom (Ehsani, 2007). Using chat has been shown to help students routinize certain often-used expressions to promote the development of automatic structure that helps develop speaking skills. This is true even if the chat is purely textual. The use of videoconferencing gives not only immediacy when communicating with a real person but also visual cues, such as facial expressions, making such communication more authentic (Stepp, 2002).

When it comes to using the computer not as a medium of communication (with other people) but as something to interact with verbally in a direct manner, the current computer technology's limitations is very noticeable. Presently, there are two fairly successful applications of automatic speech recognition (ASR) (or speech processing technology) where the computer "understands" the spoken words of the learner. The first is pronunciation training. Learners read sentences on the screen and the computer gives feedback as to the accuracy of the utterance, usually in the form of visual sound waves (Ehsani, 2007). The second is software where the learner speaks commands for the computer to do. However, speakers in these programs are limited to predetermined texts so that the computer will "understand" them (Domingo 2007).

\section{Evaluation Models}

In seeking to find out whether CALL makes a difference to students' language learning, a number of factors should be put into consideration. First of all, the environment in which the learning is taking place is determined. The environment might have been subjected to some manual form of 
conventional language learning. Therefore, the initial challenge is to determine the residual knowledge of conventional language learning of the groups that will be subjected to CALL test. This is with the aim of determining the marginal improvement that the introduction of CALL will result to. The closer the performance of the groups manual conventional language learning with each other, the better the results that will be obtained in the test.

Besides, the time frame needed for the experiment should be put into consideration. It has been observed from experience that using CALL software reduces the time frame significantly when compared with a situation in where manual conventional learning is applied. Hence, will the time spent during learning be a factor in the rating? Or will it be neglected, considering the obvious deficiency of using conventional method as far as time frame is involved. Then, how will the time frame that is required for each of the allocated group be determined?

Furthermore, what is the limit of the objectives or the criteria for judging the achievement of the objectives at the end of the learning process? This is very essential because the objectives may be tilted in favour of the CALL or in favour of the manual conventional method. Also, their might be other learning by any of the groups in the course of the test outside the scope of the objectives and so will not be captured.

Similarly considerations should be given to the proper use of CALL, as its introduction to the learning process is new technology when compared with the conventional methods in which the learners and instructors are already conversant with. Will those that will be involved in the learning process be conversant with the use of CALL software? Are the instructors sufficiently knowledgeable of 
the software and the principles behind its use? It must however be noted that new technology on the other hand can be of positive influence like increased concentration and motivation.

When attempting to compare the effectiveness of different approaches, it cannot be hoped that all the relevant variables such as time, location, culture, resources, teacher, pedagogical approach, didactical strategy, intended learning outcomes, task briefs and task outcomes can be controlled. What can be done, however, is to analyse these features carefully and systematically. The systematic evaluation can be done by recognising the influence on learning of three main perspectives (software designer, teacher and student) and taking into account three sets of interactions between them:

4 Teacher-student: a two-way direct interaction. One of the main variables here is the teacher's role, which may be 'resource provider', 'manager', 'coach', 'researcher' or 'facilitator'.

d Designer-student: primarily a one-way influence, although the designer's perception of the student's learning characteristics will implicitly be of help.

Designer-teacher: again, primarily a one-way influence, with the designer's perception of the teacher having some influence.

This framework assists the evaluator to identify the key issues on which judgements must be made in the particular context of the proposed use (predictive evaluation) or actual use (interpretive evaluation). (Soromic, 2010) 
It is clearly inadequate to consider the design of the software in isolation from its pedagogical setting. It is noted that some of the most innovative educational activities occur when teachers and students actually subvert the designer's intention, and these interactions are necessarily complex in nature. Furthermore, Anderson \& Draper (1991) assert that most times software gets used in ways not predicted by the designer, and this has significant bearing on the degree of integration of the software into the mainstream of classroom experience.

It may thus be preferable to consider how to "measure the language learning process" rather than how to "evaluate CALL" (Anderson \& Draper, 1991). There is a role for a theory of learning to predict "what factors or features of the system and the testing situation determine the measurements observed, and thus tell what measures might be worthwhile', and 'what features of an intervention to change in order to try to improve the values observed'.

\section{Role Changes for Teachers with the Use of CALL}

Although the integration of CALL into a language learning programme can lead to great anxiety among language teachers, researchers consistently claim that CALL changes, sometimes radically, the role of the teacher but does not eliminate the need for a teacher altogether (Thelmadatter, 2007). Instead of handing down knowledge to students and being the center of students' attention, teachers become guides as they construct the activities students are to do and help them as students complete the assigned tasks. In other words, instead of being directly involved in students' constructions of the language, the teacher interacts with students primarily to solve their difficulties in using the target language (grammar, vocabulary, etc.), while the students is 
given the free hand to use the language to interact with the computer and/or other people.

Elimination of a strong teacher presence has been shown to lead to larger quantity and better quality of communication such as more fluidity, more use of complex sentences and more sharing of students' personal selves. However, teacher presence is still very important to students when doing CALL activities. Teachers should be familiar enough with the resources to be used to anticipate technical problems and limitations. Students need the reassuring and motivating presence of a teacher in CALL environments. Not only are they needed during the initial learning process, they are needed to conduct review sessions to reinforce what was learned. Encouraging students to participate and offering praise are deemed important by students. Most students prefer doing work in a laboratory with a teacher's or tutor's presence to depending completely on their own.

\section{Role Changes for Students with the Use of CALL}

Students, too, need to adjust their expectations of their participation in the class in order to use CALL effectively. Rather than passively absorbing information, learners must negotiate meaning and assimilate new information through interaction and collaboration with someone other than the teacher. CALL provides students with a psychological privacy that promotes their speaking ability. It reduces the inhibitions felt in normal classroom situations and encourages the shy student to speak. The use of a language learning system encourages student to talk freely and loose their inhibitions when talking in front of their peers. Learners must also learn to interpret new information and experiences on their own terms. Moreover, shy students can feel free in their own students'-centered environment. This will raise their self- 
esteem and improve their knowledge. If students are performing collaborative project, they will do their best to perform it within set time limits. In this regard, Ravichandran (2000) noted that because the use of technology redistributes teachers' and classmates' attentions, less-able students can become more active participants in the class because class interaction is not limited to that directed by the teacher.

\section{Integrated CALL Implies 'Normalisation'}

This concept is relevant to any kind of technological innovation and refers to the stage when the technology becomes invisible, embedded in everyday practice and hence 'normalised'. To take some commonplace examples, a wristwatch, a pen, shoes, writing - are all technologies which have become normalised to the extent that we hardly even recognise them as technologies (Kigs 2008).

There is still, as Kigs points out, an element of fear, awe and exaggerated expectations surrounding CALL, and this has to be overcome in order to achieve a state of normalisation.

\section{Adaptive Hypermedia Using CALL}

These adaptive hypermedia systems, categorised by Brusilovsky (1996), have all in common that users are guided towards the paths that are considered optimal for learning. Based on the notion of what a wise teacher would normally do when presenting a question that could turn out to be too difficult to the class, the possibilities of an adaptive navigation rely on the presentation of items according to the estimated level of learners language and cognitive abilities. So, if the learner responds correctly, the next task will convey a higher level of difficulty. On the other hand, if the learner 
misses the item presented, an easier task is presented. Selection is done by the computer algorithm which adjusts the selection interactively to the successful or failed responses of the learner.

According to Waina (1990), this approach stems from the realization that we learn little about an individual's ability if we persist in asking questions that are far too difficult or far too easy for that person. We learn the most about an examinee's ability when we accurately direct our questions at the same level as the examinee's ability. Adaptive technology could consequently enhance learner's involvement in the learning process and develop autonomous access to the learning material.

Interactive Learning: Interactive in CALL means feedbacks given after learners' inputs are assessed by the system to help learners improve their language competency, which may include language skills. In addition, interactive features in CALL ensure that learning process takes place when learners engage in the lessons. Well-programmed interactive CALL lessons will provide feedbacks in terms of scores, guidelines, and customized lessons that are suitable for individual learners to move on. The designer of CALL lessons must take into considerations some language pedagogical principles which may be derived from learning theories (behaviourism, cognitive, and constructivism) and second language learning such as Krahshen's Monitor Theory. CALL is not a method. It is a tool that helps teachers to facilitate language learning process. CALL can be used to reinforce what has been learned in the classrooms. It can also be used as remedial to help learners with limited language proficiency.

Some other advantages of adaptive hypermedia for language learners could be based on: 
1. Self-Pacing: adaptive systems allow young learners to work at their own pace and act as filter to effective connotation (learners are challenged but not discouraged by the presentation of items that are far above or below in front of the class). The speed of children responses could be used as additional information for research purposes.

2. Immediate Feedback: the test can be scored immediately and provide instantaneous feedback for learners.

3. Multimedia Presentation: tests can include texts, graphics, photographs, and even full-motion video clips. The use multimedia formats on language learning can facilitate both the teaching and learning of the language.

\section{Problems and Criticisms of CALL Instruction in Language Learning}

The impact of CALL in foreign language education has been modest (Ehsani, 1998). Several reasons can be attributed to this. The first is the limitations of the technology, both in its ability and availability. There is the problem with cost (Warschauer, 2008) and the simple availability of technological resources such as the Internet (either nonexistent as can be the case in many developing countries or lack of bandwidth, as can be the case just about anywhere) (Domingo, 2007). However, the limitations that current computer technology has can be problematic as well. While computer technology has improved greatly in the last three decades, demands placed on CALL have grown even more so. One major goal is to have computers with which students can have true, human-like interaction, especially for speaking practice; however, the technology is far from that point. Not to mention that if the computer cannot evaluate a learner's speech exactly, it is almost no use at all (Ehsani, 1998). 
However, most of the problems that appear in the literature on CALL have more to do with teacher expectations and apprehensions about what computers can do for the language learner and teacher. Teachers and administrators tend to either think computers are worthless or even harmful, or can do far more than they are really capable of.

Reluctance on part of teachers can come from lack of understanding and even fear of technology. Often CALL is not implemented, even when the training has been offered to teachers. One reason for this is that from the 1960's to the 1980 's, computer technology was limited mostly to the sciences, creating a real and psychological distance for language teaching. Language teachers can be more comfortable with textbooks because it is what they are used do, and there is the idea that the use of computers threatens traditional literacy skills since such are heavily tied to books. These stem in part because there is a significant generation gap between teachers (many of whom did not grow up with computers) and students (who did grow up with them).

Also, teachers may resist because CALL activities can be more difficult to evaluate than more traditional exercises. For example, most Mexican teachers feel strongly that a completed fill-in textbook "proves" learning (Bollin, 2003). While students seem to be motivated by exercises like branching stories, adventures, puzzles or logic, these activities provide little in the way of systematic evaluation of progress.

Even teachers who may otherwise perceive benefits of CALL may be put off by the time and effort needed to implement it well. However "seductive" the power of computing systems may be, like the introduction of the audio language lab in the 1960's, those who simply expect results 
by purchasing expensive equipment are likely to be disappointed. To begin with, there are the simple matters of sorting through the numerous resources that exist and getting students ready to use computer resources. With Internet sites alone, it can be very difficult to know where to begin, and if students are unfamiliar with the resource to be used, the teacher must take time to teach it. Also, there is a lack of unified theoretical framework for designing and evaluating CALL systems as well as absence of conclusive empirical evidence for the pedagogical benefits of computers in language. Most teachers lack the time or training to create CALL-based assignments, leading to reliance on commercially-published sources, whether such are pedagogically sound or not.

\section{Conclusion}

One important fact that has emerged from this study is that language as a subject area is "different" from most other subject areas in the curriculum, namely: it is skill-based as well as knowledge-based, and in this respect it has more in common with music than, say, history or geography. The extent of influence of CALL in the quality of language learning is complex. When attempting to compare the effectiveness and influence of its use, it is not hoped that all the relevant variables can be controlled. However, a framework for evaluating the impact of it (Teacher-Student, Designer-Student and Designer-Teacher), can be of a great help in its evaluation. Finally, the positive impact of CALL generally outweighs whatever limitations it may have. This paper asserts that most of the problems of CALL usage are the reluctance and ignorance of its users. 


\section{References}

Anderson, A. \& Draper, S. An Introduction to Measuring and Understanding the Learning Process, Computers and Education, 17, 1991

Brusilovsky, $\mathrm{P}$ Methods and Techniques of Adaptive Hypermedia. User Modeling and User Adapted Interaction 6, 1996.

Domingo, N. Computer-Assisted Language Learning: Increase of freedom of submission to machines? http://www.terra.es/personal/nostat 2007

Ehsani, F. \& Eva, K. Speech Technology in Computer-Aided Language Learning: Strengths and Limitations of a New CALL Paradigm. Language Learning and Technology 2, Wikky Press, Konty 1998.

Ellis, R The study of second language acquisition. Oxford applied linguistics. OUP 2004

Hammond, M. Measuring the Impact of IT on Learning, Journal of Computer Assisted Learning, 10, 1994

Kigs Comparison of CALL Systems by Human Evaluation, http://www.morpholic.hu/public/mt/2008/compare1 2/htm, 2008

Morris, F. Child-to-child interaction and corrective feedback in a computer mediated

L2 class. Language Learning \& Technology 9, 2005.

Muegge, R Fully Automatic High Quality Machine Translation of Restricted Text: A Case Study," in Translating and the computer. Proceedings of the Twenty-Eighth International Conference on Translating and the Computer, 16-17 London, 2006

Soromic, K. Software Design: Full Consideration of Pedagogical Involvement. Newsletter Hofy. 2010 
Ten-Hacken, P. Computer-assisted language learning and the revolution in computational linguistics Linguistik online 2003

Thelmadatter, L. The Computers are Coming ... Are Here! TESOL Greece

Newsletter 95. 2007

Wainer, H (Ed.) Computerized Adaptive Testing: A Primer Hillsdale, NJ: Lawrence

Earlbaum, 1990.

Warschauer, M Computer Assisted Language Learning: An Introduction

http:/www.ict4lt.org/en/warschauerhtm. 2008.

Wintergest, A. Conceptualizing Learning Style Modalities for ESL/EFL Students. System Decapu, M.A. Vrena, 2003. 